\title{
Doenças sexualmente transmissíveis na adolescência: estudo de fatores de risco
}

\author{
Sexually transmitted diseases in adolescence: \\ study of risk factors
}

\author{
Stella R. Taquette ${ }^{1,2}$, Marília Mello de Vilhena ${ }^{2}$ \\ e Mariana Campos de Paula ${ }^{1}$
}

\begin{abstract}
RESUM0
As doenças sexualmente transmissíveis são prevalentes na adolescência e facilitadoras da contaminação pelo HIV. A baixa idade das primeiras relações sexuais, a variabilidade de parceiros, o não uso de preservativo e o uso de drogas ilícitas são apontados como fatores de risco às doenças sexualmente transmissíveis. Entrevistamos 356 adolescentes que procuraram atendimento no Núcleo de Estudos da Saúde do Adolescente da Universidade Estadual do Rio de Janeiro no período de agosto/2001 a julho/2002 com o objetivo de conhecê-los do ponto de vista da sexualidade e identificar fatores de risco às doenças sexualmente transmissíveis. Fizemos análises de freqüência e testes qui-quadrado dos dados coletados. Observamos associações estatisticamente significativas entre ter uma doenças sexualmente transmissíveis e as variáveis: atraso escolar, uso de álcool, tabaco e drogas, histórico de abuso sexual e a não utilização de preservativo nas relações sexuais. Os resultados indicam que os fatores de risco às doenças sexualmente transmissíveis na adolescência são múltiplos, sendo que o não uso do preservativo é o que tem possibilidade de redução sob a ação das equipes de saúde.
\end{abstract}

Palavras-chaves: Sexo. Adolescência. Doenças sexualmente transmissíveis-AIDS. Prevenção e controle.

\begin{abstract}
Sexually transmitted diseases are frequent in adolescence and facilitates HIV contamination. The early age limit of the first sexual intercourse, the diversity of partners, the habit of not using condoms and illicit drug abuse are pointed out as risk factors for sexual transmitted disease. We interviewed 356 adolescents who sought medical attendance at the Adolescent Health Study Center of The State University of Rio de Janeiro between August/2001 and July/2002 regarding their sexuality and to identify risk behaviors. Periodical analysis and chi-square tests were performed on the collected data. We observed statistically significant correlations between Sexual transmitted diseases and the variables of: slow learning, alcohol, tobacco and drug abuse, sexual abuse and the habit of not using condoms during sexual intercourse. The results indicate multiple Sexually transmitted disease risk behaviors in adolescence, and the failure to use condoms is the factor which is most possibly influenced by programs run by health workers.
\end{abstract}

Key-words: Sex. Adolescence. Sexually transmitted diseases-AIDS. Prevention and Control.

Na atualidade, a incidência das doenças sexualmente transmissíveis (DST) vem aumentando ${ }^{19} 20$ e pode ter por consequêencia imediata uretrites, salpingites e, a longo prazo, infertilidade, gravidez ectópica ou câncer de colo uterino ${ }^{24}$. Sabemos que ter uma DST aumenta a chance de contaminação pelo HIVe, além disso, constatamos que o perfil epidemiológico da AIDS7 mostra uma maior prevalência entre adultos jovens e uma tendência à heterossexualização e pauperização da doença ${ }^{8}$. No Brasil não há informações sobre a prevalência de DST entre adolescentes e o número de casos notificados está bem abaixo das estimativas ${ }^{16}$, talvez porque somente a AIDS e a sífilis sejam de notificação compulsória e cerca de

\footnotetext{
1. Faculdade de Ciências Médicas da Universidade do Estado do Rio de Janeiro, Rio de Janeiro, RJ. 2. Núcleo Estudos da Saúde do Adolescente, Rio de Janeiro, RJ. Endereço para correspondência: Dra Stella R. Taquette. R. Gomes Carneiro 34/802, Ipanema, 22071-110 Rio de Janeiro, RJ. Brasil.

e-mail: taquette@uerj.br

Recebido para publicação em 26/02/03

Aceito em: 20/2/2004
} 
$70 \%$ das pessoas com DST busquem tratamento em farmácias. Nos EUA, alguns autores ${ }^{29}$ inferem que a prevalência de DST entre adolescentes deve ser em torno de $25 \%$ e a faixa etária de 15 a 24 anos é a de maior risco21.

Fatores biológicos, psíquicos e sociais podem aumentar a vulnerabilidade dos adolescentes às DST. Do ponto de vista biológico, o epitélio cilíndrico do colo do útero na adolescência se encontra mais exposto e tanto as clamídeas como os gonococos têm predileção por este tecido $0^{10}$. A baixa idade da menarca pode levar a um início precoce da atividade sexual, aumentando a probabilidade de contaminação ${ }^{2}$. No âmbito psíquico, a adolescência é uma fase de definição da identidade sexual com experimentação e variabilidade de parceiros. 0 pensamento abstrato ainda incipiente nos adolescentes faz com que se sintam invulneráveis, se expondo a riscos sem prever suas consequiências. Instáveis, susceptíveis a influências grupais ${ }^{18}{ }^{13} \mathrm{e}$ familiares, estes jovens beneficiam-se de um bom relacionamento familiar para proteger-se das DST ${ }^{12}$. Na esfera social, os baixos níveis escolar e socioeconômico estão associados às DST ${ }^{28}$. Os modelos hegemônicos de comportamento de gênero também são responsáveis por atividades que colocam em risco a saúde tanto do homem quanto da mulher ${ }^{1326}$, assim como 0 uso de álcool e drogas, já comprovados por diversos estudos 369325 .

Este estudo foi feito com o objetivo de conhecer o perfil sexual dos adolescentes atendidos no Núcleo de Estudos da Saúde do Adolescente da Universidade do Estado do Rio de Janeiro (NESA) UERJ) e identificar fatores de risco às DST na adolescência.

\section{MATERIAL E MÉTODOS}

A população alvo do estudo foi o público adolescente que procurou atendimento médico no NESA/UERJ no período de agosto de 2001 ajulho de 2002. Aescolha dos participantes foi aleatória, e privilegiou-se os ambulatórios de DST, ginecologia e urologia, onde a possibilidade de existirem pacientes sexualmente ativos e portadores de DST era maior. 0 critério diagnóstico de DST foi clínico e/ou laboratorial, utilizando-se a abordagem sindrômica definida no Manual de DST do Ministério da Saúde ${ }^{15}$. Todos os participantes da pesquisa com suspeita ou diagnóstico clínico de DST foram aconselhados, no momento da consulta médica, quanto ao teste anti-HIV e outros exames laboratoriais para diagnóstico ( sorologias: sífilis e hepatite B, bacterioscopia e cultura de lesões e secreções) e, estando de acordo, estes foram realizados.

0 instrumento utilizado foi um roteiro de entrevista previamente estabelecido e testado por um estudo piloto com 20 adolescentes não incluídos na amostra do estudo. Todos os entrevistadores foram treinados antes de ir a campo. As entrevistas eram realizadas a sós com 0 adolescente, após seu consentimento e do responsável. 0 roteiro era composto de três partes. A primeira delas investigava dados pessoais, tais como: idade, situação conjugal, renda familiar, histórico escolar, uso de bebidas alcoólicas, tabaco e outras drogas. Perguntou-se sobre a frequiência à escola e escolaridade. Considerou-se atraso escolar uma defasagem maior que dois anos em relação à idade esperada para a série freqüentada. 0 uso de bebidas foi classificado em 1 vez na vida, no último mês ou seis vezes no último mês.

Na segunda parte da entrevista, indagamos ao adolescente acerca da família, das pessoas com quem morava, e de seu relacionamento com os pais, se havia, por exemplo, violência entre seus membros. Na terceira parte, 0 histórico pubertário e sexual do adolescente, a época da menarca/semenarca e da primeira relação sexual foram investigados. A respeito do primeiro coito, buscamos saber como e com quem ocorreu. Em seguida, perguntamos-lhe quanto à existência em sua vida de outras práticas sexuais, ou seja, homossexualidade, prostituição e histórico de abuso sexual. As últimas perguntas foram sobre 0 número de parceiros sexuais e sobre o uso de preservativos.

Utilizamos o teste qui-quadrado com nível de significância de $95 \%{ }^{4}$ na análise dos dados. 0 Projeto de pesquisa foi aprovado pelo Comitê de Ética em Pesquisa do Hospital Pedro Ernesto e os termos de consentimento livre e esclarecido foram assinados.

\section{RESULTADOS}

Todas as entrevistas foram realizadas em ambiente que garantia privacidade. 0 tempo médio de duração de cada uma foi de 25 minutos. Entrevistamos 356 adolescentes: destes, 109 eram sexualmente ativos portadores de DST ( Grupo A) , 115 sexualmente ativos, porém sem DST ( Grupo B) e 132 ainda não tinham iniciado atividade sexual (Grupo C). Na Tabela 1, podemos ver a composição dos grupos. Adolescentes masculinos compunham $29,5 \%$ da amostra e femininos 70,5\%. 0 predomínio de moças ocorreu devido ao grande volume de atendimento do ambulatório de ginecologia. 0 percentual de homens e mulheres em cada grupo foi semelhante.

Tabela 1 - Distribuição dos participantes segundo a presença de atividade sexual e doenças sexualmente transmisśiveis.

\begin{tabular}{|c|c|c|c|c|c|c|c|c|}
\hline \multirow{3}{*}{ Sexo } & \multirow{2}{*}{\multicolumn{2}{|c|}{ Amostra total }} & \multirow{2}{*}{\multicolumn{2}{|c|}{$\begin{array}{c}\text { Grupo A } \\
\text { sexual/ativos c/DST }\end{array}$}} & \multirow{2}{*}{\multicolumn{2}{|c|}{$\begin{array}{c}\text { Grupo B } \\
\text { sexual/ativos s/DST }\end{array}$}} & \multirow{2}{*}{\multicolumn{2}{|c|}{$\begin{array}{c}\text { Grupo C } \\
\text { s/atividade sexual }\end{array}$}} \\
\hline & & & & & & & & \\
\hline & $\overline{n^{0}}$ & $\%$ & $n^{0}$ & $\%$ & $n^{0}$ & $\%$ & $n^{0}$ & $\%$ \\
\hline$\overline{\text { Masculino }}$ & 105 & 29,5 & 31 & 28,4 & 32 & 27,8 & 42 & 31,8 \\
\hline Feminin & 251 & 70,5 & 18 & 71,6 & 83 & 72,2 & 90 & 68,2 \\
\hline otal & 356 & 100,0 & 109 & 100,0 & 115 & 100,0 & 132 & 100,0 \\
\hline
\end{tabular}

A faixa etária dos adolescentes variou de 12 a 19 anos, sendo a média de idade nos grupos de portadores de DST ( grupo A) e não portadores (grupo B + grupo C) de 16,8 e 16,3 anos respectivamente. Arenda familiar média foi de 3,6 salários mínimos no grupo com DST e de 4,3 no grupo sem DST. Na situação conjugal, a maioria dos adolescentes era solteira. Em relação à escolaridade, grande parte dos adolescentes estava na escola. Entretanto, 0 atraso escolar maior que dois anos foi encontrado com mais frequiência entre os portadores de DST, sendo esta associação estatisticamente significativa. Estes dados estão na Tabela 2.

As variáveis uso de tabaco, bebidas alcoólicas e drogas ilícitas tiveram uma associação estatisticamente significativa com ser 
Tabela 2 - Distribuição dos adolescentes segundo algumas características demográficas.

\begin{tabular}{|c|c|c|c|c|}
\hline \multirow[t]{2}{*}{ Item pesquisado } & \multicolumn{2}{|c|}{$\begin{array}{c}\text { Portadores de DST } \\
\text { Grupo A } \\
\end{array}$} & \multicolumn{2}{|c|}{$\begin{array}{c}\text { Năo portadores de DST } \\
\text { Grupo B+C } \\
\end{array}$} \\
\hline & $n^{0}$ & $\%$ & $\mathrm{n}^{0}$ & $\%$ \\
\hline Idade média (anos) & 16,8 & & 16,3 & \\
\hline Renda familiar ( sal min) & 3,6 & & 4,3 & \\
\hline \multicolumn{5}{|l|}{ Situação conjugal } \\
\hline sem companheiro/a & 95 & 87,2 & 233 & 94,3 \\
\hline com companheiro/a & 14 & 12,8 & 14 & 5,7 \\
\hline \multicolumn{5}{|l|}{ Histórico escolar } \\
\hline estão na escola & 82 & 75,2 & 217 & 87,9 \\
\hline atraso escolar>2anos & $53^{*}$ & 48,6 & 62 & 25,1 \\
\hline
\end{tabular}

portador de DST. Entre os portadores de DST os percentuais foram de $22 \%, 10,1 \%$ e $20,2 \%$ de uso de tabaco, de bebidas alcoólicas seis vezes ou mais no último mês e de outras drogas ilícitas, respectivamente. E entre os não portadores os percentuais das mesmas variáveis foram de 7,7\%, 3,6\% e 4\%. 0 uso de tabaco ocorreu em $22 \%$ e 7,7\% dos portadores de DST e não portadores respectivamente. Estes dados podem ser visualizados na Tabela 3.

Tabela 3 - Distribuição dos adolescentes segundo o uso de tabaco, álcool e drogas.

\begin{tabular}{|c|c|c|c|c|}
\hline \multirow[t]{2}{*}{ Item pesquisado } & \multicolumn{2}{|c|}{$\begin{array}{c}\text { Portadores de DST } \\
\text { Grupo A }\end{array}$} & \multicolumn{2}{|c|}{$\begin{array}{c}\text { Năo portadores de DST } \\
\text { Grupo } B+C\end{array}$} \\
\hline & $n^{0}$ & $\%$ & $n^{0}$ & $\%$ \\
\hline & 109 & 100,0 & 247 & 100,0 \\
\hline \multicolumn{5}{|l|}{ Uso de tabaco } \\
\hline $\operatorname{sim}$ & $24^{*}$ & 22,0 & 19 & 7,7 \\
\hline não & 85 & 78,0 & 228 & 92,3 \\
\hline \multicolumn{5}{|l|}{ Uso de bebidas alcoólicas } \\
\hline nunca usou & 40 & 36,7 & 91 & 36,8 \\
\hline usou 1x na vida & 19 & 17,4 & 91 & 36,8 \\
\hline usou no último mês & 39 & 35,8 & 56 & 22,7 \\
\hline usou $6 \mathrm{x}$ ou mais no último mês & $11 *$ & 10,1 & 9 & 3,7 \\
\hline \multicolumn{5}{|l|}{ Uso de outras drogas } \\
\hline sim & $22^{*}$ & 20,2 & 10 & 4,0 \\
\hline não & 87 & 79,8 & 237 & 96,0 \\
\hline
\end{tabular}

Os dados sobre a família e sobre 0 início pubertário podem ser visualizados na Tabela 4. As famílias biparentais e 0 relacionamento bom entre os pais foram significativamente mais freqüentes entre os adolescentes não portadores de DST do que nos portadores. A violência intrafamiliar foi relatada por $51,4 \%$ dos portadores de DST e por $36,4 \%$ dos não portadores. A idade média da menarca/semenarca foi semelhante nos dois grupos. 0 histórico de abuso sexual foi significativamente mais freqüente entre os adolescentes com DST do que entre os sem DST.

No inquérito sobre atividade sexual constatamos que do total dos entrevistados ( $n=356$ ), 224 já tinham tido intercursos sexuais. Na Tabela 5, estão descritas as principais características do histórico sexual dos grupos de sexualmente ativos. A idade média da primeira relação sexual foi de 14,7 anos entre os portadores de DST (Grupo A) e de 15 anos entre os sexualmente ativos não portadores de DST (Grupo B) . Grande parcela dos adolescentes iniciou a atividade sexual antes dos 15 anos, porém não houve uma associação estatisticamente significativa entre a baixa idade do primeiro coito e ter uma DST. Houve relato de gravidez anterior em 27,5\% do Grupo A e 17,4\% do Grupo B. A prática homossexual foi referida por poucos e cerca de $10 \%$ dos adolescentes do Grupo Ajá tinham se prostituído, assim como $3,5 \%$ do Grupo B. 0 número de parceiros foi maior do que dois em 29,4\% do Grupo A e 33\% do Grupo B. Em relação ao uso do preservativo, observou-se uma relação estatisticamente significativa entre a freqüência às vezes ou nunca e ser portador de DST.

Os diagnósticos mais comuns foram as uretrites entre os homens e as vulvovaginites entre as mulheres. As primeiras foram confirmadas com cultura da secreção uretral. Nas vulvovaginites o diagnóstico foi predominantemente clínico.

Tabela 4 - Distribuição dos adolescentes segundo dados familiares e pubertários.

\begin{tabular}{|c|c|c|c|c|}
\hline \multirow[t]{2}{*}{ Item pesquisado } & \multicolumn{2}{|c|}{$\begin{array}{c}\text { Portadores de DST } \\
\text { Grupo A }\end{array}$} & \multicolumn{2}{|c|}{$\begin{array}{c}\text { Năo portadores de DST } \\
\text { Grupo } B+C\end{array}$} \\
\hline & $\mathrm{n}^{0}$ & $\%$ & $n^{0}$ & $\%$ \\
\hline & 109 & 100,0 & 247 & 100,0 \\
\hline Famílias biparentais & 30 & 27,5 & $141^{*}$ & 57,1 \\
\hline Relacionamento bom entre os pais & 33 & 30,5 & $150 *$ & 60,7 \\
\hline Violência intrafamiliar & 56 & 51,4 & 90 & 69,4 \\
\hline Histórico de abuso sexual & $28^{*}$ & 25,7 & 24 & 9,7 \\
\hline Idade média men/semenarca (anos) & 12,4 & & 11,8 & \\
\hline
\end{tabular}

Tabela 5 - Distribuição dos adolescentes segundo as características da atividade sexual.

\begin{tabular}{|c|c|c|c|c|}
\hline \multirow[t]{2}{*}{ Item pesquisado } & \multicolumn{2}{|c|}{$\begin{array}{c}\text { Portadores de DST } \\
\text { Grupo A } \\
\end{array}$} & \multicolumn{2}{|c|}{$\begin{array}{c}\text { Năo portadores de DST } \\
\text { Grupo B }\end{array}$} \\
\hline & $\mathrm{n}^{0}$ & $\%$ & $n^{0}$ & $\%$ \\
\hline & 109 & 100,0 & $\mathrm{n}^{\circ} 115$ & $\% 100,0$ \\
\hline \multicolumn{5}{|l|}{ Características do $1^{\circ}$ coito } \\
\hline idade média (anos) & 14,7 & & 15 & \\
\hline ocorreu antes dos 15 anos & 51 & 46,8 & 45 & 39,1 \\
\hline \multicolumn{5}{|l|}{ Como ocorreu $01^{\circ}$ coito } \\
\hline espontâneo & 103 & 94,5 & 110 & 95,7 \\
\hline forçado & 1 & 0,9 & 0 & 0 \\
\hline por pressão & 5 & 4,6 & 5 & 4,3 \\
\hline \multicolumn{5}{|l|}{ Com quem ocorreu $01^{\circ}$ coito } \\
\hline namorado/a & 73 & 67,0 & 92 & 80,0 \\
\hline amigo/a & 34 & 31,2 & 16 & 13,9 \\
\hline profissional do sexo & 2 & 1,9 & 5 & 4,3 \\
\hline marido & 0 & 0 & 2 & 1,8 \\
\hline Histórico de gravidez & 30 & 27,5 & 20 & 17,4 \\
\hline Homossexualidade & 7 & 6,4 & 6 & 5,2 \\
\hline Histórico de prostituição & 11 & 10,1 & 4 & 3,5 \\
\hline Parceiros $\left(n^{0}>2\right)$ & 53 & 48,6 & 38 & 33,0 \\
\hline \multicolumn{5}{|l|}{ Uso de preservativo } \\
\hline nunca ou às vezes & $85^{*}$ & 78,0 & 56 & 48,7 \\
\hline sempre ou quase sempre & 24 & 22,0 & 59 & 51,3 \\
\hline
\end{tabular}


Não foi possível a coleta de secreção vaginal ou testes ( $\mathrm{pH}$ e aminas) em todos os casos. 0 diagnóstico da sífilis foi feito por história, sinais e sintomas clínicos e por exame VDRL (Venereal Disease Research Laboratory). Nos casos de HPV (papilomavirus humano) o diagnóstico foi clínico, havendo lesões condilomatosas, e do herpes genital pela presença de úlceras genitais rasas, dolorosas, precedidas de lesões vesiculares. Houve quatro casos de HIV+, por transmissão sexual, com diagnóstico sorológico realizado obedecendose ao fluxograma do Ministério da Saúde ${ }^{15}$ num adolescente masculino e em três femininos. Verificamos um caso de hepatite B num rapaz que tinha sido tratado de uma uretrite gonocócica e que apresentou IgM positiva ( Anti-HbcIgM) nos exames sorológicos realizados, sem histórico de uso de injeções contaminadas ou transfusões sangüíneas. Houve três casos de escabiose em que o diagnóstico foi feito através dos sintomas clínicos (escoriações e sulcos genitais pruriginosos) e teste terapêutico, após terem sido afastados outros diagnósticos através de exames laboratoriais. Em alguns casos houve concomitância de mais de uma DST. Na Tabela 6, optouse por descrever somente a distribuição dos diagnósticos principais de DST.

Tabela 6 - Distribuição dos diagnósticos das DST em homens e mulheres..

\begin{tabular}{|c|c|c|c|c|}
\hline \multirow[b]{2}{*}{ Diagnóstico principal } & \multicolumn{2}{|c|}{ Homens } & \multicolumn{2}{|c|}{ Mulheres } \\
\hline & $\mathrm{n}^{0}$ & $\%$ & $\mathrm{n}^{0}$ & $\%$ \\
\hline & 31 & 100,0 & 78 & 100,0 \\
\hline Uretrites gonocócicas e não gonocócicas & 17 & 54,8 & 3 & 3,8 \\
\hline Vulvovaginites ( candidíase/vaginose/tricomoníase) & 0 & 0 & 47 & 60,3 \\
\hline $\mathrm{HPV}$ & 7 & 22,6 & 15 & 19,2 \\
\hline Sífilis & 3 & 9,7 & 7 & 9,0 \\
\hline HIV & 1 & 3,2 & 3 & 3,8 \\
\hline Herpes genital & 0 & 0 & 2 & 2,6 \\
\hline Escabiose & 2 & 6,5 & 1 & 1,3 \\
\hline Hepatite B & 1 & 3,2 & 0 & 0 \\
\hline
\end{tabular}

\section{DISCUSSÃ0}

A baixa idade das primeiras relações sexuais e a variabilidade de parceiros, citados na literatura científica como fatores de risco às DST, não se confirmaram em nossa amostra. Apesar da baixa idade média tanto da menarca/ semenarca como do primeiro intercurso sexual no grupo estudado, idade essa inferior à média de outros estudos sobre sexualidade na adolescência ${ }^{27}$, nenhum destes fatores apresentou uma relação estatisticamente significativa com ter uma DST. Outra informação que pode ser verificada na bibliografia médica ${ }^{10}{ }^{18}$ e também nos meios de comunicação leiga é a de que o jovem é um ser promíscuo, pois a adolescência é uma fase da vida de experimentação sexual. Não foi 0 que se observou neste estudo. Menos da metade $(31,2 \%)$ do total de adolescentes sexualmente ativos referiu já ter tido mais de dois parceiros sexuais e esta variabilidade não se apresentou como um fator de risco às DST. 0 diagnóstico de DST mais freqüente foi o de vulvovaginite entre as moças e de uretrite entre os rapazes, dado semelhante ao encontrado por outros autores brasileiros ${ }^{11}$.

0 uso infreqüente do preservativo, 0 atraso escolar e 0 uso de drogas lícitas e ilícitas foram as principais variáveis associadas às DST neste estudo. Para se obter uma diminuiç̧ão destes riscos são necessários investimentos estruturais em nossa sociedade, principalmente no que diz respeito ao acesso universal à educação e saúde. Os adolescentes não podem e não devem ficar fora da escola. Em relação ao uso de drogas, 0 meio social de modo geral e principalmente pais e educadores devem dar 0 exemplo e ser menos tolerantes em relação a seu uso e abuso, sem, contudo, apelar para atitudes policialescas, punitivas, etc. Quanto ao preservativo, campanhas de incentivo à sua utilização em todas as relações sexuais precisam ser intensificadas. Esta é a principal tarefa a ser abraçada pelas equipes de saúde que trabalham com adolescentes. No Brasil o preservativo é muito pouco utilizado, principalmente entre os jovens. Segundo dados do Ministério da Saúde ${ }^{14}$, os mais baixos índices de uso (em torno de 0,2 a 1,4\%) se encontram na faixa etária de 15 a 19 anos. Nos países desenvolvidos, em especial a França, os programas de saúde realizados no sentido de reduzir 0 risco de infecção pelo HIV provocaram uma mudança profunda na sexualidade da juventude. Houve um aumento notável da utilização do preservativo, especialmente no início da vida sexual. No ano de 1993, 75\% dos jovens de 15 a 18 anos tiveram sua primeira relação sexual com camisinha, sendo que em 1985 este percentual fora de $7 \%{ }^{22}$.

Os adolescentes em geral sabem que o preservativo evita doenças e gravidez, mas mesmo assim não 0 usam. Existe uma enorme lacuna entre 0 nível de conhecimento e 0 uso efetivo da camisinha. A juventude aponta numerosas justificativas para não usá-la: esquecimento, custos e desprazer na relação sexual. Neste estudo o principal problema identificado, ou seja, o uso infreqüente do preservativo pode ser combatido pelas equipes de saúde e assim tornar possível uma diminuição dos índices de DST na adolescência, e, conseqüentemente da infecção pelo HIV. Para os pesquisadores um caminho efetivo talvez seja associar a camisinha ao prazer resultante da segurança que ela proporciona. Não usá-la significa correr riscos de engravidar sem querer e/ou sem poder e de ficar doente ou até morrer. Pensamos que a tranqüilidade e garantia que 0 preservativo traz pode resultar em um ganho semelhante ao que a pílula anticoncepcional trouxe em seu surgimento. Não devemos, porém, abandonar outras medidas de redução do risco de contaminação por DST-AIDS igualmente importantes: orientações sobre 0 início da vida sexual, fidelidade mútua, redução do número de parceiros e abandono de práticas sexuais de risco. Urge que estratégias eficazes sejam criadas para se alcançar este objetivo e, para tal, os adolescentes têm de ser ouvidos enquanto participantes do processo. De nada adianta oferecer-lhes soluções prontas. 


\section{AGRADECIMENTOS}

Agradecemos a participação, durante 0 desenvolvimento da pesquisa, da médica ginecologista Dra. Renata Bessa de Andrade, da enfermeira Rejane Araújo de Souza e dos seguintes alunos bolsistas de graduação da Universidade Estadual do Rio de Janeiro: Felipe Kaezer dos Santos, Felipe Nirenberg, Ene Garcez Neto e Úrsula Pérsia Paulo dos Santos.

\section{REFERÊNCIAS BIBLIOGRÁFICAS}

1. Aberastury A, Knobel M. Adolescência normal. Porto Alegre, Artes Médicas, 1988.

2. Adih WK, Alexander CS. Determinants of condom use to prevent HIV infection among youths in Ghana. Journal of Adolescent Health 24: 63-72, 1999.

3. Anteghini M, Fonseca H, Ireland M, Blum RW. Health risk behaviors and associated risk and protective factors among Brazilian Adolescents in Santos, Brazil. Journal of Adolescent Health 28: 295-302, 2001.

4. Arango HG. Bioestatística. Rio de Janeiro, Guanabara Koogan, 2001.

5. Bayley SL, Pollock MPH, Martin CS, Lynch K. Risky sexual behaviors among adolescents with alcohol use disorders. Journal of Adolescent Health 25: 179-181, 1999 .

6. Bastos Fl, Carlini-Contrin B. 0 consumo de substâncias psicoativas entre os jovens brasileiros: dados, danos \& algumas propostas. In: Comissão Nacional de População e Desenvolvimento (CNPD) Jovens Acontecendo na Trilha das Políticas Públicas, Editora CNPD, Brasília, p. 645-669, 1998.

7. Castilho E, Szwarcwakd CL. Mais uma pedra no meio do caminho dos jovens brasileiros: a AIDS. In: Comissão Nacional de População e Desenvolvimento (CNPD) Jovens Acontecendo na Trilha das Políticas Públicas, Editora CNPD, Brasília, p.197-207, 1998.

8. Chequer P. A Aids no Brasil: Perfil epidemiológico e ações. A Folha Médica $117: 1-2,1998$.

9. Cox JM, D'AngeloV, Silber TJ. Substance abuse and syphilis in urban adolescents; a new risk factor for an old disease. Journal of Adolescent Health 13: 483-486, 1992.

10. Grant LM. Adolescent sexuality. Pediatric Clinics of North America 35: 1271-1289, 1988.

11. Isolan TB, Carvalho AVV, Almeida Filho GL. Perfil do atendimento ao adolescente no setor de doenças sexualmente transmissíveis da Universidade Federal Fluminense. Jornal Brasileiro de Doenças Sexualmente Transmissíveis 13: 9-30, 2001.

12. Karofsky PS, Zeng L, Kosorok MR. Relationship between adolescent-parental communication and initiation of first intercourse by adolescents. Journal of Adolescent Health 28: 41-45, 2000.
13. Magnusson C. Adolescent girls' sexual attitudes and opposite-sex relations in 1970 and in 1996. Journal of Adolescent Health 28: 242-252, 2001.

14. Ministério da Saúde. Preservativo masculino. Secretaria de Assistência à Saúde. Programa Nacional de DST e AIDS, MS, Brasília, 1997.

15. Ministério da Saúde. Manual de controle de Doenças Sexualmente Transmissíveis. Brasília. Coordenação Nacional de DST e AIDS. Secretaria de Políticas de Saúde, MS, Brasília, 1999.

16. Ministério da Saúde. Dados e Pesquisas em DST e Aids, <http:// www.aids.gov.br, 27 novembro, MS, Brasília, 2002.

17. Mota MP. Gênero e sexualidade: fragmentos de identidade masculina nos tempos de AIDS. Cadernos de Saúde Pública 14: 145-155, 1998.

18. Murphy DA, Durako SJ, Moscicki AB, Vermund SH, Ma Y, Schwarz DF, Muenz LR. No change in health risk behaviors over time among HIV infected adolescents in care: role of psychological distress. Journal of Adolescent Health 29S: 57-63, 2001.

19. Petri V. Doenças de Transmissão Sexual. In: Comissão de Saúde do Adolescente, Adolescência e Saúde, Secretaria Estadual, São Paulo, Paris Editorial, p. 183-190, 1988.

20. Ramos C, May S. Aspectos históricos das doenças sexualmente transmissíveis. In: Secretaria Municipal de Saúde do Rio de Janeiro ( org) Saúde em Foco, Rio de Janeiro, Editora Secretaria Municipal, RJ, p. 5-11, 1998.

21. Richert CA. A method for identifying persons at high risk for sexually transmitted infections: opportunity for targeting intervention. American Journal of Public Health 83:520-524, 1993.

22. Rudelic-Fernandez D. A Sexualidade dos Jovens em Tempos de AIDS: Atos e Falas. In: Borillo D, Fédida P, Fraisse G, Gayon J, Matlock J, Rudelic-Fernandez (eds) A Sexualidade tem futuro? Edições Loyola, São Paulo, p. 55-72, 2002.

23. Scivoletto S, Tsuji RK, Abdo CHN, Queiroz S, Andrade AG, Gattaz WF. Relação entre consumo de drogas e comportamento sexual de estudantes de $2^{\circ}$ grau de São Paulo. Revista Brasileira de Psiquiatria 21: 87-94, 1999.

24. Silber TJ, D'Angelo L, Woodward K. El SIDA y otras enfermedades de transmissión sexual. In: OPAS ( org) La salud del adolescente y del joven, Organización Panamericana de la Salud, p. 273-284, 1995.

25. Tapert SF, Aarons G, Sedlar G, Brown SA. Adolescente substance use and sexual risk-taking behavior. Journal of Adolescent Health 28: 181-189, 2001.

26. Villela W. "Homem que é homem também pega AIDS?" In: Arilha M, Ridenti GU, Medrado B (eds) Homens e Masculinidades. Outras Palavras, Editora 34, São Paulo, SP, p. 129-142, 1988.

27. Warren CW, Kann L, Small ML, Santelli JS, Collins JL, Kolbe LJ. Age of initiating selected health-risk behaviors among high school students in the United States. Journal of Adolescent Health 21: 225-231, 1997.

28. Waystaff DA, Delameth JD, Havens KK. Subsequent infection among adolescent african-american males attending a sexually transmitted disease clinic. Journal of Adolescent Health 25: 217-226, 1999.

29. Yaber WL, Parillo AV. Adolescents and sexually transmitted diseases. Journal of School Health 2: 331-238, 1992. 